\title{
Kooperation zwischen Krankenhäusern und Universitäten in der Umsetzung der berufsqualifizierenden Tätigkeit III
}

\author{
Ulrich Schweiger \\ Klinik für Psychiatrie und Psychotherapie, Helios Hanseklinikum Stralsund, Stralsund, Deutschland
}

\section{Schlüsselwörter \\ Approbationsordnung für Psychotherapeutinnen und Psychotherapeuten - Berufsqualifizierende Tätigkeit III - Entrustable Professional Activities (auf zentrale Kompetenzen fokussiertes Ausbildungskonzept)}

\section{Zusammenfassung}

Der Regierungsentwurf einer Approbationsordnung für Psychotherapeutinnen und Psychotherapeuten definiert eine berufsqualifizierende Tätigkeit III. Der Text beschreibt Möglichkeiten und Schwierigkeiten der Umsetzung dieses neuen Elements im Studium Psychotherapie.

(c) 2020 S. Karger AG, Basel

Cooperation between Hospitals and Universities in the Implementation of Training Elements during the Master Degree Program "Psychotherapy"

\section{Keywords}

Licensing for clinical psychologists in Germany .

Practical training during the master degree program

"psychotherapy" · Entrustable professional activities

\begin{abstract}
The new government regulations on licensing for clinical psychologists in Germany define the requirements for practical training during the master degree program "psychotherapy." The text describes the scope and challenges of implementation of this new training element.
\end{abstract}

(c) 2020 S. Karger AG, Basel

\section{Einleitung}

Das Psychotherapeutengesetz vom 15. November 2019 hat die Ausbildung, die zum Beruf der Psychotherapeutin und des Psychotherapeuten führt, reformiert und auf eine neue Rechtsgrundlage gestellt. Anders als bisher wird die Approbation als Psychotherapeutin oder Psychotherapeut künftig nicht mehr nach einer postgradualen Ausbildung erteilt, sondern nach dem erfolgreichen Abschluss eines Bachelor- und eines Masterstudiums sowie nach dem Bestehen der psychotherapeutischen Prüfung.

Zum Gesetz wurde am 20. Dezember 2019 ein Regierungsentwurf einer Approbationsordnung für Psychotherapeutinnen und Psychotherapeuten veröffentlicht (Verordnung des Bundesministeriums für Gesundheit, 
Drucksache 670/19). Im folgenden Text soll es um die berufsqualifizierende Tätigkeit III und Möglichkeiten deren attraktiver Umsetzung gehen.

Im $\$ 18$ dieser Verordnung heißt es:

Berufsqualifizierende Tätigkeit III - angewandte Praxis der Psychotherapie

(1) Die berufsqualifizierende Tätigkeit III - angewandte Praxis der Psychotherapie dient der Vertiefung der praktischen Kompetenzen in der psychotherapeutischen Versorgung.

(2) Die studierenden Personen sind während der berufsqualifizierenden Tätigkeit III -angewandte Praxis der Psychotherapie zu befähigen, die Inhalte, die sie in der hochschulischen Lehre während der berufsqualifizierenden Tätigkeit II - vertiefte Praxis der Psychotherapie erworben haben, in realen Behandlungssettings und im direkten Kontakt mit Patientinnen und Patienten umzusetzen. Hierzu sind sie unter Anwendung der wissenschaftlich geprüften und anerkannten psychotherapeutischen Verfahren und Methoden an der Diagnostik und der Behandlung von Patientinnen und Patienten zu beteiligen, indem sie

1 aufbauend auf wissenschaftlich fundierten Kenntnissen zu psychischen Funktionen, Störungen und diagnostischen Grundlagen mittels wissenschaftlich geprüfter Methoden Anamnesen und psychodiagnostischeUntersuchungen beimindestens 10 Patientinnen und Patienten verschiedener Alters- und Patientengruppen aus mindestens 4 verschiedenen Störungsbereichen mit jeweils unterschiedlichen Schwere- und Beeinträchtigungsgraden durchführen, die mindestens die folgenden Leistungen umfassen:

(a) 4 Erstgespräche,

(b) 4 Anamnesen, die per Video aufzuzeichnen und von den studierenden Personen schriftlich zu protokollieren sind,

(c) 4 wissenschaftlich fundierte psychodiagnostische Untersuchungen,

(d) 4 Indikationsstellungen oder Risiko- und Prognoseeinschätzungen einschließlich Suizidalitätsabklärung und

(e) 4 Patientenaufklärungen über diagnostische und klassifikatorische Befunde,

2 an mindestens einer psychotherapeutischen ambulanten Patientenbehandlung im Umfang von mindestens 12 aufeinanderfolgenden Behandlungsstunden teilnehmen, die unter Verknüpfung von klinischpraktischen Aspekten mit ihren jeweiligen wissenschaftlichen Grundlagen durchgeführt wird und $\mathrm{zu}$ der begleitend diagnostische und therapeutische Handlungen eingeübt werden,

3 an mindestens 2 weiteren einzelpsychotherapeutischen Patientenbehandlungen, bei denen eine Patientin oder ein Patient entweder ein Kind oder eine Ju- gendliche oder ein Jugendlicher sein soll, mit unterschiedlicher Indikationsstellung im Umfang von insgesamt mindestens 12 Behandlungsstunden teilnehmen und dabei die Diagnostik, die Anamnese und die Therapieplanung übernehmen sowie die Zwischen- und Abschlussevaluierung durchführen,

4 mindestens 3 verschiedene psychotherapeutische $\mathrm{Ba}$ sismaßnahmen wie Entspannungsverfahren, Psychoedukation oder Informationsgespräche mit Angehörigen selbständig, aber unter Anleitung durchführen,

5 Gespräche mit bedeutsamen Bezugspersonen bei mindestens 4 Patientenbehandlungen führen und dokumentieren,

6 mindestens 12 gruppenpsychotherapeutische Sitzungen begleiten,

7 selbständig und eigenverantwortlich mindestens ein ausführliches psychologisch-psychotherapeutisches Gutachten erstellen, das ausschließlich Ausbildungszwecken dienen darf, und

8 an einrichtungsinternen Fortbildungen teilnehmen.

(3) Für die berufsqualifizierende Tätigkeit III - angewandte Praxis der Psychotherapie sind 20 ECTS-Punkte (European Credit Transfer System) zu vergeben.

(4) Von dem entsprechenden Arbeitsaufwand von 600 $\mathrm{h}$ entfallen:

1450 h Präsenzzeit in Form von mindestens sechswöchigen studienbegleitenden Übungspraktika auf die stationäre oder teilstationäre Versorgung und

$2150 \mathrm{~h}$ auf die ambulante Versorgung mit Präsenzzeit während laufender Therapien sowie während diagnostisch-gutachterlicher Datenerhebungen.

(5) Die berufsqualifizierende Tätigkeit III - angewandte Praxis der Psychotherapie findet in Hochschulambulanzen, Einrichtungen der psychotherapeutischen, psychiatrischen, psychosomatischen, neuropsychologischen Versorgung oder in interdisziplinären Behandlungszentren mit Psychotherapieschwerpunkt statt.

\section{Hintergründe}

Wir erleben zurzeit einen enormen Zuwachs von gesundheitsrelevantem Wissen im Bereich der Verhaltenswissenschaften sowie der Vielfalt und empirischen Fundierung psychotherapeutischer Methoden. Hieraus ergibt sich die Notwendigkeit, einen Beruf zu entwickeln, der das Wissen der Behavioral Sciences und spezifisch der Psychotherapie für die Gesundheit der Menschen nutzbar macht. Die Bedeutung psychologischer und verhaltensbezogener Interventionen hat auch in der gesamten Heilkunde enorm zugenommen. Psychologische und verhaltensbezogene Therapien in der Medizin stützen sich auf eine große Menge von Evidenz und sind für viele Erkrankungen die Behandlung erster Wahl. Zentral für 
die berufliche Rolle des Psychotherapeuten der Zukunft ist deshalb, dass er das vorhandene verhaltenswissenschaftliche und therapeutische Wissen in praktische Interventionen umsetzen kann, in ähnlicher Weise, wie ein Arzt biomedizinisches Wissen umsetzt.

Es handelt sich bei der Reform nicht nur um strukturelle Veränderungen an der Ausbildung und Weiterbildung, sondern um eine Transformation der Rolle von Psychotherapeuten. Sie werden zu unabhängigen Akteuren auf Augenhöhe mit den anderen selbständigen Heilkundeberufen. Mit diesem neuen Status sind auch neue Erwartungen verbunden: Psychotherapeuten können sich nicht mehr darauf beschränken, Experten in der Anwendung einer bestimmten Psychotherapiemethode zu sein. Sie brauchen eine umfassende Kompetenz über den gesamten Bereich der Psychotherapie und ihrer Anwendung bei einem breiten Spektrum von Patienten. Weiterhin brauchen sie Schnittstellenkompetenz, Teamkompetenz und die Bereitschaft, in der Behandlung schwerkranker Patienten mitzuarbeiten. Auf einer organisatorischen Ebene ist eine intensive Kooperation zwischen Psychotherapie und Medizin in der Umsetzung der Approbationsordnung gefordert.

Eine weitere und für die berufsqualifizierende Tätigkeit III zentrale Erwartung ist, dass am Ende des Studiums der Psychotherapie, ähnlich wie bei jungen Ärztinnen und Ärzten, ein Repertoire an Entrustable Professional Activities vorhanden ist [Shorey et al., 2019]. Dabei geht es um basale Handlungskompetenzen, die für den Arbeitsbereich typisch sind und die Berufsanfängern in der auf das Studium folgenden Weiterbildung in den ersten Arbeitstagen abverlangt werden. Die unter $\$ 18(2)$ aufgeführten Inhalte entsprechen diesem Konzept. Es geht allgemein gesprochen um folgende Kompetenzen: ein Erstgespräch mit einem Patienten zu führen, dabei Gefährdungsmomente zu erkennen, eine Arbeitsdiagnose zu bilden, die Befunde schriftlich zu dokumentieren, mit der untersuchten Patientin, der leitenden Psychologin oder der Oberärztin über die Befunde zu kommunizieren, sowie zu beginnen, sich unter Supervision in die Bereiche Einzeltherapie und Gruppentherapie einzuarbeiten.

\section{Umsetzbarkeit}

Es gibt zur Zeit einen hohen Bedarf an akademischem Personal im Gesundheitsbereich. Praktika während des Studiums sind für Kliniken eine ideale Gelegenheit, mögliche zukünftige Mitarbeiterinnen und Mitarbeiter kennenzulernen. Es ist deshalb damit zu rechnen, dass viele Kliniken sich bemühen werden, entsprechende Plätze anzubieten. Im praktischen Jahr des Medizinstudiums gibt es einen ausgeprägten Wettbewerb um die Studenten. Die geplante Praktikumszeit von 12 Wochen ist zwar etwas geringer als die viermonatigen Abschnitte im praktischen Jahr, aber lange genug, um Studenten für unterstützende Aufgaben sinnvoll einzusetzen. Ausbildung explizit in die Versorgung zu integrieren kann auch für Krankenhäuser, die ihren Schwerpunkt im Bereich der Versorgung sehen, attraktiv sein. Ein Krankenhaus, das von Studentinnen als Ausbildungsstätte betrachtet wird, gewinnt auch aus Patientensicht an Renommee. Integration von Ausbildung in die Versorgung ist auch tatsächlich geeignet, die Prozessqualität zu verbessern.

Eine Barriere stellen allerdings die hohen Anforderungen dar. Eine Klinik, die entsprechende Praktikumsstellen anbietet, braucht approbierte psychologische Psychotherapeutinnen oder Fachärztinnen für Psychiatrie und Psychotherapie, Kinder- und Jugendlichenpsychiatrie und Psychotherapie oder psychosomatische Medizin und Psychotherapie, die bereit und befähigt sind, Studentinnen und Studenten anzuleiten und im Erwerb der Entrustable Professional Activities zu supervidieren. Zwar könnten Teile des obengenannten Anforderungskatalogs auch im Rahmen der ambulanten Praktika bzw. an Hochschulambulanzen im Rahmen der universitären Lehre absolviert werden, trotzdem wird die Hauptverantwortung zur Erfüllung dieser Auflagen schwerpunktmäBig in die Hand der Kliniken gelegt. Die Kliniken brauchen hierfür eine funktionierende Weiterbildungsstruktur mit allen hierfür notwendigen Elementen. Spezifische Anforderungen an diese Personen und Strukturen sind in der Approbationsordnung zwar nicht spezifiziert, müssen aber von den jeweiligen Universitäten entwickelt und im Dialog mit den Praktikumsstellen implementiert werden. Der zusätzliche Zeitaufwand der supervidierenden Therapeutinnen für die Betreuung der Praktikanten ist erheblich. Die Supervisorinnen müssen für die Untersuchungen und Interventionen mit Vor- und Nachbereiten zum Teil selbst zugegen sein, Videoaufzeichnungen ansehen und Protokolle auf ihre Richtigkeit überprüfen. Viele der Praktikantinnen werden an Kliniken arbeiten, die nicht von Forschung und Lehre geprägt sind, sondern ihren Versorgungsauftrag an erster Stelle sehen. Die Vorgaben des BTQ III lassen es nicht zu, dass die Praktikanten einfach mitlaufen und "learning by doing" oder "observational learning" im Vordergrund steht. Die supervidierenden Therapeutinnen brauchen ein didaktisches Konzept und müssen dieses umsetzen.

Für die Umsetzbarkeit ist auch wichtig, dass die Kliniken gegebenenfalls ihr Dienstleistungsangebot prüfen und unter Umständen auch neue, durch die Praktikanten zu erbringende Leistungen anbieten, die zum Vorteil von Patientinnen und Patienten, ihren Angehörigen und damit auch der Klinik und der Psychotherapeutenausbildung sind. Ausführlichere Informationsgespräche für Patienten und ihre Angehörigen zu Krankheiten und Behandlungs- 
konzepten, zusätzliche psychodiagnostische Untersuchungen, aber auch weitere Maßnahmen im Bereich der Qualitätssicherung oder einer "wissenschaftsbasierten Praxis" sind Beispiele hierfür. Im therapeutischen Bereich ist auch an durch Praktikanten begleitete Aktivitäten im lebensnahen Kontext zu denken. Soweit die Entfernungen es zulassen, können Beobachtungen aus der Lebensumwelt eingeholt und therapeutische Aufgaben im Kontext von Hausbesuchen durchgeführt werden. PsychotherapiePraktikanten können durch eine Tätigkeit als aktive Therapie-Coaches die Behandlung bereichern. Es wäre naheliegend, wenn Kliniken für ihre betreuenden Mitarbeiter und für die Psychotherapie-Praktikanten schriftliche Manuale für die Praktikumszeit erstellen, um dadurch Anleitung zu geben und Transparenz zu schaffen.

\section{Zusammenfassung und Diskussion}

Die neue Approbationsordnung möchte sicherstellen, dass Psychotherapeutinnen beim Einstieg in den Beruf über ein ausreichendes Repertoire an Basiskompetenzen verfügen. Hierzu werden für die berufsqualifizierende Tätigkeit III eine Vielzahl von spezifischen Anforderungen formuliert. Diese Anforderungen sind grundsätzlich sinnhaft, es besteht jedoch die Gefahr, dass hierdurch Barrieren entstehen, welche die Verfügbarkeit von Praktikumsplätzen einschränken. Die Universitäten und die kooperierenden medizinischen Einrichtungen sind ge- fordert, die Anforderungen so zu spezifizieren, dass die berufsqualifizierende Tätigkeit in für alle Seiten praktikabler Weise umgesetzt werden kann. Funktionierende Kooperationen zwischen den medizinischen Einrichtungen und den in Psychotherapie ausbildenden Universitäten sind der Schlüssel zur Umsetzung der Approbationsordnung.

\section{Statement of Ethics}

Da für den Artikel keinerlei personenbezogene oder andere Daten ausgewertet wurden, war keine Genehmigung durch eine Ethikkommission oder Studienregistrierung erforderlich.

\section{Disclosure Statement}

Der Autor ist zweiter Vorstand des Deutschen Fachverbands für Verhaltenstherapie (DVT).

\section{Funding Sources}

Bezüglich des Artikels erfolgte keine finanzielle Unterstützung durch Dritte.

Literatur

Shorey S, Lau TC, Lau ST, Ang E. Entrustable professional activities in health care education: a scoping review. Med Educ. 2019 Aug;53(8): 766-77. 